\title{
Resonating with Others: The Effects of Self-Construal Type on Motor Cortical Output
}

\author{
Sukhvinder S. Obhi, ${ }^{1,2}$ Jeremy Hogeveen, ${ }^{1}$ and Alvaro Pascual-Leone ${ }^{3}$ \\ ${ }^{1}$ Centre for Cognitive Neuroscience and Department of Psychology, Wilfrid Laurier University, Waterloo, Ontario, Canada N2L 3C5, ${ }^{2}$ Institute of Cognitive \\ Neuroscience, University College London, London WC1N 3AR, United Kingdom, and 'Berenson-Allen Center for Noninvasive Brain Stimulation, Beth \\ Israel Deaconess Medical Center, and Department of Neurology, Harvard Medical School, Boston, Massachusetts 02215
}

\begin{abstract}
"Self-construal" refers to how individuals view and make meaning of the self, and at least two subtypes have been identified. Interdependent self-construal is a view of the self that includes relationships with others, and independent self-construal is a view of the self that does not include relations with others. It has been suggested that priming these two types of self-construal affects the cognitive processing style that an individual adopts, especially with regard to context sensitivity. Specifically, an interdependent self-construal is thought to promote attention to others and social context to a greater degree than an independent self-construal. To investigate this assertion, we elicited motor-evoked potentials with transcranial magnetic stimulation during an action observation task in which human participants were presented with either interdependent or independent self-construal prime words. Priming interdependent self-construal increased motor cortical output whereas priming independent self-construal did not, compared with a no-priming baseline condition. These effects, likely mediated by changes in the mirror system, essentially tune the individual to, or shield the individual from, social input. Interestingly, the pattern of these self-construal-induced changes in the motor system corroborates with previously observed selfconstrual effects on overt behavioral mimicry in social settings, and as such, our results provide strong evidence that motor resonance likely mediates nonconscious mimicry in social settings. Finally, these self-construal effects may lead to the development of interventions for disorders of deficient or excessive social influence, like certain autism spectrum and compulsive imitative disorders.
\end{abstract}

\section{Introduction}

In their classic paper, Markus and Kitiyama (1991) suggested that individual concepts of self, or self-construals, vary across cultures (for review, see Markus and Kitiyama, 1999; Cross et al., 2011; Chiao et al., 2009; Han and Northoff, 2009). Specifically, Markus and Kitiyama (1991) reported that, in collectivist cultures, individuals tend to adopt an interdependent self-construal that includes relationships with others, whereas in individualist cultures, individuals tend to adopt an independent self-construal that does not include relationships with others. For example, in a collectivist culture such as Japanese society, people tend to define themselves as fundamentally interconnected with significant others and in terms of group affiliation (e.g., father, daughter, coworker, etc.). In contrast, typical members of individualist cultures, such as American society, tend to define themselves with respect to stable internal characteristics (e.g., friendly, compassionate, ambitious, etc.) (Cross et al., 2011). While much early

Received June 23, 2011; revised July 30, 2011; accepted Aug. 16, 2011.

Author contributions: S.O. and J.H. designed research; J.H. performed research; S.O. and J.H. analyzed data; S.O., J.H., and A.P.L. wrote the paper.

This work was supported by a Natural Sciences and Engineering Research Council of Canada Discovery Grant and Ontario Early Research Award (S.O.). We thank Roham Farzami for assistance with the preparation of the figures and Dr. Derek Mitchell for useful discussion relating to this paper.

The authors declare no competing financial interests.

Correspondence should be addressed to Sukhvinder Obhi, Centre for Cognitive Neuroscience, Wilfrid Laurier University, Waterloo, Ontario, Canada N2L 3C5. E-mail: sobhi@wlu.ca.

DOI:10.1523/JNEUROSCI.3186-11.2011

Copyright $\odot 2011$ the authors $\quad 0270-6474 / 11 / 3114531-05 \$ 15.00 / 0$ work was performed in the cross-cultural context, it has also become evident that different forms of self-construal are represented within an individual and within a culture, and that situational determinants can activate one or the other form (Brewer and Gardner, 1996; Chiao et al., 2009; Cross et al., 2011). This allows for experimental priming of self-construal and for studying how different forms of self-construal affect behavior and brain activity.

Several social psychological studies have investigated the effects of self-construal type on social cognition. For example, Haberstroh et al. (2002) showed that Western participants who were primed with interdependent self-construal were more likely to take on a target person's perspective than participants who were primed with independent self-construal. Similarly, individuals primed with interdependent self-construal listed more possible arguments of a debating opponent, and listened more to the other person's point of view, than those primed with an independent self-construal (Gardner and Seeley, 2001). Differences in nonsocial cognition have also been reported. For example, Kuhnen et al. (2000) showed that Western participants primed with independent self-construal were faster at detecting geometric figures embedded in larger geometric designs than participants primed with interdependent self-construal. Furthermore, Lin et al. (2008) demonstrated that priming independent self-construal promoted greater focus on individual elements in a global-local processing task, whereas priming interdependent self-construal promoted greater focus on the global target. Together, the evidence to date suggests that interdependent self-construal pro- 
motes a higher degree of context sensitivity than independent self-construal. If this is true, then self-construal priming should also affect the degree to which a person processes observed actions of another person, with interdependent primes facilitating processing relative to independent primes.

The aim of the current study was to determine the effects of self-construal priming on the human motor cortex while participants engaged in an action observation task. We predicted that interdependent priming would facilitate the processing of observed actions by increasing motor resonance indexed by motor-evoked potentials, whereas independent priming would reduce motor resonance. This pattern of results would demonstrate a strong effect of self-construal type on the activity of primary motor cortex and thereby provide direct evidence that self-construal priming modulates brain activity.

\section{Materials and Methods}

Participants. Ten participants (five male, five female; age range, $18-39$ years) took part in the experiment. All participants had normal or corrected-to-normal vision and all were righthanded. The experiment conformed to local ethical guidelines, participants were screened for contraindications before participation, and informed consent was obtained from all participants. Subjects received monetary compensation for their participation.

Apparatus and stimuli. The experiment was programmed using Superlab v.4 (Cedrus) and run on a Dell desktop computer with stimuli displayed on a 20 inch LCD monitor. Transcranial magnetic stimulation (TMS) was performed with a taped figure-eight coil attached to a Magstim Rapid ${ }^{2}$ system. EMG data were recorded using a psychophysiological record-

ing system (Biopac Systems). Digital pulses were sent from the computer running Superlab to the Biopac system, which in turn was used to trigger TMS pulses through a solid-state relay drive. Motor-evoked potentials (MEPs) were measured with pairs of $8 \mathrm{~mm}$ surface electrodes placed in a belly-tendon arrangement over the abductor pollicis brevis (APB) muscle of the participant's right hand. A ground was placed on the ulnar styloid of the right wrist. The EMG signal was acquired with a $5 \mathrm{kHz}$ sampling rate, amplified (to $5.0 \mathrm{mV}$ ), filtered (bandpass, $10-500 \mathrm{~Hz}$ ), and sent to a laptop computer for off-line analysis.

Experimental stimuli were videos depicting a right hand (palm facing down) squeezing a rubber ball between the thumb and index finger such that the rubber ball was substantially deformed. The videos consisted of a single squeeze repeated five, six, seven, eight, or nine times. Self-construal primes were superimposed in black font throughout some of the videos (Fig. 1b). A list of 30 self-construal primes ( 15 independent, 15 interdependent) was adapted with permission from previous studies (Kuhnen and Hannover, 2000). The prime words were as follows: Interdependent primes: together, integrate, help, others, family, social, give, lives, team, connected, accommodate, friend, group, cooperate, companion, relationship, affiliation, community, dependence, alliance; Independent primes: independent, individuality, unique, autonomous, distinct, life, distinguished, myself, singular, unconnected, apart, solo, lone, oneself, alone, special, mine, solitary, differ, self. In a pre-experiment baseline condition, 30 MEPs were recorded from the APB muscle while participants passively observed a fixation cross (Fig. 1a). As reported previously, a lexical decision task revealed no fundamental difference in lexical access times for these two word lists (Hogeveen and Obhi, 2010). Video-editing was performed using Final Cut Express v.4 (Apple). All inferential statistical analysis was performed using SPSS Statistics 18.0 (SPSS).

Procedure. Participants were seated with their right arm flexed at $90^{\circ}$ and resting on a pillow positioned on the desk in front of the computer monitor (Fig. 1c), and a cardboard box and black fabric were used to occlude vision of their hand. The experimenter located the vertex using the inion-nasion line and the preauricular points at the posterior end of each zygomatic arch as landmarks. TMS was delivered through a figureeight coil, held normal to the scalp and $45^{\circ}$ to midline, with current flowing in a posterior-anterior direction over the left primary motor cortex. Stimulation began at $70 \%$ stimulator output and the coil was moved incrementally until the site eliciting the greatest MEP in the right APB muscle was identified. The optimal location was marked on a Lycra swim cap worn by participants, and a mechanical arm was used to hold the coil in a fixed position. Once the coil was in position, stimulator output was decreased in $5 \%$ intervals until the lowest intensity sufficient to elicit five MEPs of $\sim 1 \mathrm{mV}$ peak-to-peak amplitude out of $10 \mathrm{TMS}$ pulses was identified. For the sample of participants used in this experiment, stimulation intensity ranged from $56 \%$ to $70 \%$ (mean: $64.1 \%$ ) of stimulator output. 
Before the main experiment, the baseline level of motor cortical output was assessed by delivering 30 TMS pulses at $5 \mathrm{~s}$ intervals while participants passively viewed a fixation cross (Fig. 1a). After the baseline block, each participant was exposed to three randomized blocks of 75 trials showing a rubber ball being squeezed. Specifically, blocks were comprised of 25 neutral (no prime), 25 interdependent prime, and 25 independent prime trials. Each block contained 30 TMS pulses, which were delivered at points of maximum flexion during the action alone (10), interdependent self-construal (10), and independent self-construal (10) videos (i.e., 30 pulses total per condition). Participants were instructed to count the number of squeezes contained in each video and report the number verbally at the end of each trial, to ensure attention to the action.

Following the TMS session, participants were asked whether they noticed anything about the types of words presented in the experiment. After responding, the hypotheses, purpose, and methods of the study were explained in a debriefing session.

\section{Results}

Mean peak-to-peak MEP amplitude was calculated for each experimental condition. MEP amplitudes that were greater or less than 3 SDs away from the condition mean were excluded from analysis (resulted in the removal of $5 \%$ of the MEP data). After outlier exclusion, mean peak-to-peak MEP amplitude for each experimental condition was entered into a repeated-measures ANOVA, which revealed a main effect of condition $\left(F_{(3,27)}=\right.$ 9.474, $p<0.001$, partial $\eta^{2}=0.513$; interdependent prime condition, independent prime condition, no-prime condition, and fixation cross baseline). Planned $t$ tests confirmed that nopriming and interdependent priming conditions produced MEPs of significantly greater amplitude than the MEPs in the fixation cross baseline condition (fixation vs no priming: $t_{(9)}=3.79, p=$ 0.002; fixation vs interdependent priming: $t_{(9)}=4.12, p=$ 0.0015 ) and that the independent priming condition produced an almost significant increase in MEPs compared with the fixation cross baseline (fixation vs independent priming: $t_{(9)}=1.605$, $p=0.0715$ ). Thus, observation of the videos, regardless of the priming condition, led to a clear facilitation of the MEPs compared with the baseline fixation-cross condition (Fig. 2a). Figure $2 b$ shows five representative MEPs from a single subject for the interdependent, independent, and no-priming conditions. Such facilitation of motor cortical outputs associated with action observation has been reported previously (Fadiga et al., 1995, 2005; Strafella and Paus, 2000; Maeda et al., 2002), and neurophysiologic and neuroimaging studies in humans suggest that it is mediated by the mirror neuron system (Buccino et al., 2004; Binkofski and Buccino, 2006) in a manner similar to what is observed in nonhuman primates (Gallese et al., 1996; Rizzolatti et al., 2008).

To compare the effects of interdependent and independent self-construal priming on the excitability of motor cortical output, we calculated the percentage change in MEP amplitude in each priming condition from the no-priming (i.e., action alone) condition. A repeated-measures ANOVA revealed that interdependent and independent self-construal priming produced significantly different percentage changes in MEP amplitude (percentage change for interdependent self-construal priming $=$ $16 \%$, percentage change for independent self-construal priming $\left.=-11 \% ; F_{(1,9)}=16.133, p=0.003\right)$. Thus, while interdependent self-construal priming facilitated motor cortical outputs beyond the facilitation induced by unprimed action observation, independent self-construal priming relatively suppressed the facilitation induced by unprimed action observation, although this reduction below baseline levels was not quite significant.

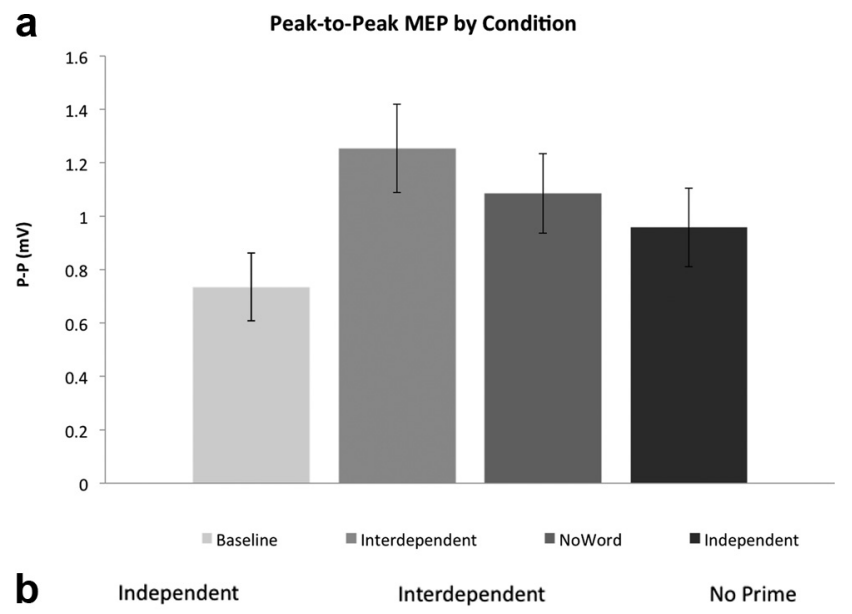

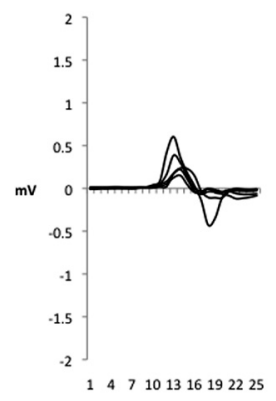

ms

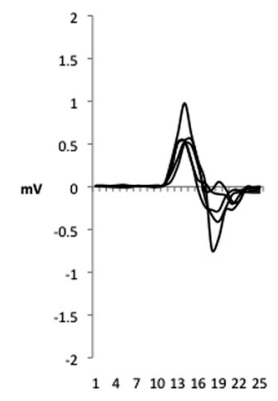

$\mathrm{ms}$

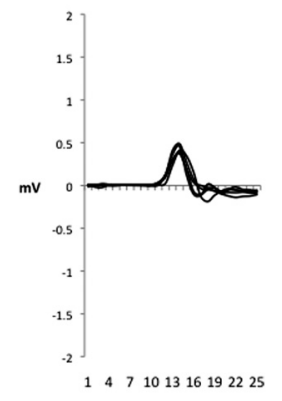

$\mathrm{ms}$
Figure 2. a, Mean MEPs for each experimental condition. Note that the mean MEP for the interdependent condition was significantly different from the mean MEP for the independent condition. See the Results section for statistics. Error bars are SEM. P-P, Peak-to-peak. $\boldsymbol{b}$, Five representative MEPs from a single subject for the independent prime condition, interdependent prime condition, and the no prime condition. Time scale is from 15 to $40 \mathrm{~ms}$ post-TMS stimulus (indicated here as $1-25 \mathrm{~ms}$ ).

\section{Discussion}

The aim of this experiment was to determine the effects of selfconstrual priming on motor cortical activation during passive action observation. Participants observed a video of a model squeezing a rubber ball between their index finger and thumb. On $40 \%$ of trials, MEPs in the APB muscle were elicited by focal TMS delivered to the contralateral motor cortex. On each trial, an interdependent prime, an independent prime, or no prime was superimposed over the video. Results show that self-construal priming induces dynamic changes in motor corticospinal excitability during action observation, even in mixed blocks of trials wherein interdependent and independent self-construal primes (and no-prime trials) are pseudorandomly interleaved. This reveals a rapid capacity of the brain to switch between self-construal reference frames (i.e., on a timescale of seconds) and provides novel insights into the effects of these shifts on underlying neural processing in the motor cortex.

The pattern of data we observed is suggestive of a dynamic hyperactivation of motor-resonant mechanisms in response to interdependent self-construal priming and a hypoactivation of motor-resonant mechanisms in response to independent selfconstrual priming (although independent priming only showed a trend toward a significant reduction in motor cortical excitability compared with a no-priming condition).

Our results also corroborate important findings from social psychology, where it has been shown that, when individuals are primed with an interdependent self-construal, they tend to 
mimic an interaction partner more in a subsequent social interaction than when they are primed with an independent selfconstrual (van Baaren et al., 2003). Behavioral mimicry in social settings has crucial downstream effects, such as increased prosocial behavior (Chartrand and van Baaren, 2009), and some researchers have suggested that mimicry acts like a social glue that brings people together and maximizes the chances of successful social outcomes (Lakin and Chartrand, 2003). In addition to the effect of self-construal on behavioral mimicry, other research has suggested changes in processing style, resulting from changes in the scope of visual attention and sensitivity to context, as a result of interdependent or independent self-construal perspective. However, to date there has been very little attempt to address the mechanisms underlying these self-construal effects (Lin et al., 2008; Lieberman and Forster, 2009). Our study is among the first to address this gap in our collective knowledge.

Given the essentially parallel pattern of self-construal effects on motor cortical output (shown here) and actual behavioral mimicry (van Baaren et al., 2003), our findings provide some of the most direct evidence to date that motor resonance, possibly induced via a human mirror system, mediates the occurrence of behavioral mimicry in social settings (van Baaren et al., 2003). While several authors have suggested a link between the mirror system and social phenomena such as behavioral mimicry (Chartrand and van Baaren, 2009; Iacoboni, 2009; Obhi and Hogeveen, 2010), until now, no direct evidence supporting this notion has been provided. To be clear, the fact that the same moderator (i.e., self-construal) produces parallel patterns of results on motor cortical output and mimicry in social settings supports the idea that the two phenomena are mediated by common mechanisms (although this has not been proven).

What mechanisms might account for the observed selfconstrual-induced modulation of motor cortical outputs? Given the nature of the primes used in this experiment, it seems sensible that any explanation of our results should be based on some form of top-down modulation of activity in brain areas that produce motor resonance. One possibility is that interdependent selfconstrual priming induces greater activity in brain areas sensitive to (social) visual motor input and independent priming induces a reduction in activation in these same areas. Because we are hypothesizing a modulation of areas involved in the initial coding of social action input, this explanation can be classed as an input modulation account of self-construal priming. The exact mechanisms involved in this input-modulation cannot be gleaned from the present work. Again, this idea predicts greater activation in higher-order visual areas (that are thought to provide input to the parietofrontal mirror network), such as the posterior part of the superior temporal sulcus (pSTS), when interdependence is primed, and lower activation when independence is primed. This prediction is consistent with psychophysiological data showing a modulation in higher-order visual processing with interdependent and independent self-construal priming (Lin et al., 2008). Furthermore, such changes in activity in higher-order visual areas should be correlated with activity in areas implicated in selfrelated cognition, such as the medial prefrontal cortex (mPFC). This suggestion is supported by previous studies in which topdown modulation of higher-order social visual processing has been demonstrated and explained with the idea that MPFC [and temporoparietal junction (TPJ)] directly modulates processing in STS (Teufel et al., 2010). Future studies examining whether interdependent priming modulates activity in the pSTS seem warranted.
Another type of account posits that some aspect of mirror activity itself is affected by the primes. This type of account could be labeled a resonance-modulation account of self-construal effects and may or may not occur in conjunction with the input modulation described above. Several studies have shown that increased self-focus is correlated with increased brain activity in self-related processing regions, including the medial prefrontal and other cortical midline structures (CMS) (Northoff et al., 2006; Han and Northoff, 2009). However, as has been discussed in the literature, the exact nature of the self-related processing that $\mathrm{mPFC}$ and CMS activity represents is varied and complex, and is thought to include processes related to the other self-other distinction. (Uddin et al., 2005, 2007). One possibility is that increased activity in self-related brain areas due to independent self-construal priming could increase any inhibitory effect of these areas on regions involved in processing information pertaining to others. Interdependent self-construal priming might reduce activity in self-related brain areas, which would in turn reduce any normally inhibitory influence of these areas on regions that process information emanating from others in the context. Evidence for an inhibitory relationship between areas involved in self-related processing and mirror areas comes from observations that mPFC activity is negatively correlated with activity in the insula, an area implicated in mirroring observed action (Cheng et al., 2007; Spengler et al., 2010). Cheng et al. (2007) argued for an inhibitory effect of the mPFC on the insula, which they suggest helps reduce the automatic mirroring that occurs when someone watches another individual in pain.

An additional, perhaps simpler, mechanism is a direct influence from medial prefrontal areas to premotor cortex that modulates motor cortical output (Miller, 2000; Amodio and Frith, 2006). This idea could be described as a direct-modulation account of self-construal effects.

Finally, in the present task, participants did not produce an action in response to the video. Hence, perhaps inhibition of automatic imitation was necessary in all trials. Spengler et al. (2009) have suggested that prefrontal areas (anterior frontal median cortex) and the TPJ are activated when shared representations have to be controlled to prevent automatic imitation. If MEPs index the success of imitative control processes (larger MEPs, less successful control; smaller MEPs, more successful control), then self-construal priming could be described as modulating imitative control. According to Spengler et al. (2009), this ability is fundamentally linked to the ability to distinguish self from other. A specific prediction is that interdependence serves to reduce the self-other distinction, whereas independence serves to increase it [and these changes result in corresponding changes in motor cortical output (Brass et al., 2009)]. Future work addressing these input modulation, resonance modulation, and direct modulation possibilities is required.

Given the relationship between mirror system activity and empathy (Avenanti et al., 2005; Fecteau et al., 2008; Keysers and Gazzola, 2009), rapid modulations of mirror sensitivity could have explanatory power for certain social psychological phenomena. For example, in the famous Milgram obedience to authority experiments (Milgram, 1965), the majority of teachers were prepared to deliver fatal shocks to learners when they were in separate rooms, but this proportion was reduced when an interdependent mental frame was facilitated by placing teacher and learner in close proximity (for evidence of a link between physical proximity and interdependent self-construal, see Holland et al., 2004). The applied significance of our findings could also include 
beneficial effects for individuals with autism spectrum disorders, who have been suggested to have deficits in mirror processing (Iacoboni and Dapretto, 2006; Ramachandran and Oberman, 2006). One possibility is that inducing interdependent selfconstrual in such individuals could make them more responsive to contextual social information emanating from other individuals. Conversely, the effects of independent self-construal priming could be harnessed to reduce the responsiveness of the mirror system such that individuals with disorders such as compulsive imitation might benefit. Furthermore, individuals with psychopathic personality traits have been shown to be hyper-responsive (at the sensorimotor resonance level) to other peoples' pain (Fecteau et al., 2008). Thus, self-construal priming may also have a place in remedial programs for such individuals. Nonclinical applications include the possibility to make learning by observation more efficient by inducing interdependent self-construal.

In summary, inducing interdependent or independent selfconstrual differentially modulates motor cortical output on a timescale of seconds. Whereas interdependent self-construal seems to induce hyperactivity in the motor resonant system, independent self-construal shows a pattern suggestive of induced hypoactivity. Together with other results from social psychology, our results support the idea that motor resonant systems in the human brain mediate behavioral mimicry. In this way, we provide some of the most direct evidence to date supporting the suggested causal link between motor resonance and behavioral mimicry (Chartrand and Bargh, 1999). Our findings could also be clinically relevant for the development of novel therapeutic interventions for disorders of excessive or reduced social influence.

\section{References}

Amodio DM, Frith CD (2006) A meeting of minds: the medial frontal cortex and social cognition. Nat Rev Neurosci 7:268-277.

Avenanti A, Bueti D, Galati G, Aglioti SM (2005) Transcranial magnetic stimulation highlights the sensorimotor side of empathy for pain. Nat Neurosci 8:955-960.

Binkofski F, Buccino G (2006) The role of ventral premotor cortex in action execution and action understanding. J Physiol Paris 99:396-405.

Brass M, Ruby P, Spengler S (2009) Inhibition of imitative behaviour and social cognition. Philos Trans R Soc Lond B Biol Sci 364:2359-2367.

Brewer MB, Gardner W (1996) Who is this "we"? Levels of collective identity and self representations. J Pers Soc Psychol 71:83-93.

Buccino G, Binkofski F, Riggio L (2004) The mirror neuron system and action recognition. Brain Lang 89:370-376.

Cheng Y, Lin CP, Liu HL, Hsu YY, Lim KE, Hung D, Decety J (2007) Expertise modulates the perception of pain in others. Curr Biol 17:1708-1713.

Chiao JY, Harada T, Komeda H, Li Z, Mano Y, Saito D, Parrish TB, Sadato N, Iidaka T (2009) Neural basis of individualistic and collectivistic views of self. Hum Brain Mapp 30:2813-2820.

Chiao JY, Harada T, Komeda H, Li Z, Mano Y, Saito D, Parrish TB, Sadato N, Iidaka T (2010) Dynamic cultural influences on neural representations of the self. J Cogn Neurosci 22:1-11.

Cross SE, Hardin EE, Gercek-Swing B (2011) The what, how, why, and where of self-construal. Pers Soc Psychol Rev 15:142-179.

Fadiga L, Fogassi L, Pavesi G, Rizzolatti G (1995) Motor facilitation during action observation: a magnetic stimulation study. J Neurophysiol 73: $2608-2611$.

Fadiga L, Craighero L, Olivier E (2005) Human motor cortex excitability during the perception of others' action. Curr Opin Neurobiol 15:213218.

Fecteau S, Pascual-Leone A, Théoret H (2008) Psychopathy and the mirror neuron system: preliminary findings from a non-psychiatric sample. Psychiatry Res 160:137-144.

Gallese V, Fadiga L, Fogassi L, Rizzolatti G (1996) Action recognition in the premotor cortex. Brain 119:593-609.

Gardner WL, Seeley EA (2001) Confucius, "Jen," and the benevolent use of power. In: The use and abuse of power (Lee-Chai AY, Bargh JA, eds), pp 263-280. Philadelphia: Psychology.

Haberstroh S, Oyserman D, Schwarz N, Kühnen U, Ji LJ (2002) Is the interdependent self more sensitive to question context than the independent self? Self-construal and the observation of conversational norms. J Exp Soc Psychol 38:323-329.

Han S, Northoff G (2009) Understanding the self: a cultural neuroscience approach. Prog Brain Res 178:203-212.

Holland RW, Roeder UR, van Baaren RB, Brandt AC, Hannover B (2004) Don't stand so close to me: the effects of self-construal on interpersonal closeness. Psychol Sci 15:237-242.

Iacoboni M (2009) Imitation, empathy, and mirror neurons. Annu Rev Psychol 60:653-670.

Iacoboni M, Dapretto M (2006) The mirror neuron system and the consequences of its dysfunction. Nat Rev Neurosci 7:942-951.

Keysers C, Gazzola V (2009) Expanding the mirror: vicarious activity for actions, emotions, and sensations. Curr Opin Neurobiol 19:666-671.

Kühnen U, Hannover B (2000) Assimilation and contrast in social comparisons as a consequence of self-construal activation. Eur J Soc Psychol 30:799-811.

Lin Z, Lin Y, Han S (2008) Self-construal priming modulates visual activity underlying global/local perception. Biol Psychol 77:93-97.

Maeda F, Kleiner-Fisman G, Pascual-Leone A (2002) Motor facilitation while observing hand actions: specificity of the effect and role of observer's orientation. J Neurophysiol 87:1329-1335.

Markus HR, Kitayama S (1999) Culture and the self: implications for cognition, emotion, and motivation. New York: Psychology.

Milgram S (1965) Some conditions of obedience and disobedience to authority. Hum Relat 18:57-76.

Miller EK (2000) The prefrontal cortex and cognitive control. Nat Rev Neurosci 1:59-65.

Northoff G, Heinzel A, de Greck M, Bermpohl F, Dobrowolny H, Panksepp J (2006) Self-referential processing in our brain: a meta-analysis of imaging studies on the self. Neuroimage 31:440-457.

Obhi SS, Hogeveen J (2010) Incidental action observation modulates muscles activity. Exp Brain Res 203:427-435.

Ramachandran VS, Oberman LM (2006) Broken mirrors: a theory of autism. Sci Am 295:62-69.

Rizzolatti G, Fabbri-Destro M (2008) The mirror system and its role in social cognition. Curr Opin Neurobiol 18:179-184.

Spengler S, von Cramon DY, Brass M (2009) Control of shared representations relies on key processes involved in mental state attribution. Hum Brain Mapp 30:3704-3718.

Spengler S, Brass M, Kühn S, Schütz-Bosbach S (2010) Minimizing motor mimicry by myself: self-focus enhances online action-control mechanisms during motor contagion. Conscious Cogn 19:98-106.

Strafella AP, Paus T (2000) Modulation of cortical excitability during action observation: a transcranial magnetic stimulation study. Neuroreport 11:2289-2292.

Teufel C, Fletcher PC, Davis G (2010) Seeing other minds: attributed mental states influence perception. Trends Cogn Sci 14:376-382.

Uddin LQ, Kaplan JT, Molnar-Szakacs I, Zaidel E, Iacoboni M (2005) Self face recognition activates a frontoparietal mirror network in the right hemisphere: an event related fMRI study. Neuroimage 25:926-935.

Uddin LQ, Iacoboni M, Lange C, Keenan JP (2007) The self and social cognition: the role of cortical midline structures and mirror neurons. Trends Cogn Sci 11:153-157.

van Baaren RB, Maddux WW, Chartrand TL, de Bouter C, van Knippenberg A (2003) It takes two to mimic: behavioral consequences of selfconstruals. J Pers Soc Psychol 84:1093-1102. 